\title{
Observational Report
}

\section{e Conversion of Chronic Pain Patients from Full- Opioid Agonists to Sublingual Buprenorphine}

\author{
Jonathan Daitch, MD, Michael Frey, MD, David Silver, PharmD, Carol Mitnick, ARNP, \\ Danielle Daitch, BA, and Joseph Pergolizzi Jr., MD
}

From: IAdvanced Pain

Management and Spine Specialists, Fort Myers, FL

For complete author affiliations, see page 66 .

Address correspondence: Jonathan Daitch, MD

8255 College Parkway Suite 200

Fort Myers, FL 33919 E-mail:

jdaitchmd@gmail.com

Disclaimer: There was no external funding in the preparation of this manuscript.

Conflict of interest: Dr. Daitch is a paid speaker for Reckitt Benckiser.

Manuscript received: 05/09/2011

Revised manuscript received: 12/002/2011 Accepted for publication: o1/30/2012

Free full manuscript: www.painphysicianjournal. com
Background: Sublingual buprenorphine-naloxone (buprenorphine SL) is a preparation that is used to treat opioid dependence. In addition, the Drug Enforcement Administration (DEA) has acknowledged the legality of an off-label use to treat pain with a sublingual buprenorphine preparation. Buprenorphine SL is unique among the opioid class of analgesics; this compound has a high affinity for the mu-receptor, yet only partially activates it. Thus, buprenorphine SL can provide analgesia, yet minimize opioid side effects. Many patients on high doses of traditional opioid medication develop tolerance. Despite escalating medication dosage, a subset of patients had a paradoxical increase in pain, which has been characterized as opioid-induced hyperalgesia $(\mathrm{OIH})$. Buprenorphine SL, on the other hand, may even be anti-hyperalgesic and may have utility in treating these challenging patients.

Objective: To determine the effectiveness of converting patients from traditional full agonist opioid medication to sublingual buprenorphine, as well as to identify patient groups that are most likely to benefit from this therapy. Patients who underwent conversion either had developed tolerance with diminished analgesia or were experiencing side effects on their opioid medications.

Study Design: An observational report of outcomes assessment.

Setting: An interventional pain management practice setting in the United States.

Methods: Retrospective data from clinical records was compiled on 104 de-identified chronic pain patients whose personal information had been redacted (60 men and 44 women, aged 21-78) and who had previously been treated with opioid-agonist drugs; they were converted to buprenorphine SL in tablet form during the study. Chronic pain was defined as persistent pain for at least 6 months. Data collected from patient profiles included age, sex, diagnosis, medication history, pre-induction opioid intake, reason for detoxification, preinduction Clinical Opiate Withdrawal Score (COWS), and if applicable, cause of buprenorphine SL cessation. Pain levels and Quality of Life scores were recorded before and after conversion to buprenorphine SL.

Outcome Measures: Level of analgesia for patients who continued conversion to sublingual buprenorphine for more than 2 months.

Results: After initiation of buprenorphine SL therapy for more than 2 months, the mean pain scores on a scale from 0-10 decreased by 2.3 points $(P<0.001)$. Patient Quality of Life (QoL scale) was not significantly affected by buprenorphine SL therapy $(P=0.14)$. The success rate was highest for patients using morphine, oxycodone, and fentanyl before buprenorphine SL induction. These patient groups had a 3.7 point decrease in pain for those taking morphine, a 2.5 point decrease in pain for those taking oxycodone, and a 2.2 point decrease for those taking fentanyl. The smallest pain reduction was seen in the patient group using oxymorphone before conversion with a 1.1 point decrease in pain. Patients taking between 100-199 mg morphine equivalent per day experienced the greatest reduction (2.7 points) in pain scores. Patients taking between 200 and $299 \mathrm{mg}$ morphine equivalent before buprenorphine SL induction exhibited a decrease of over 2 points on average. Patients taking $>400 \mathrm{mg}$ morphine equivalent reported the smallest reduction in pain scores, on average a 1.1 point decrease.

Limitations: This study is limited by its observational nature.

Conclusions: Patients continuing buprenorphine SL therapy for more than 60 days reported significant decreases in pain (2.3 points). Patients on doses of opioid medication between 100-199 mg morphine equivalents seemed to fare better with conversion to buprenorphine SL than patients on the highest doses (> $400 \mathrm{mg}$ morphine equivalents). The opioid drug used by the patient before buprenorphine SL induction appears to have some effect on buprenorphine SL conversion success. Patients previously taking morphine, oxycodone, and fentanyl had the greatest decrease in pain after conversion to buprenorphine SL.

Key Words: Sublingual buprenorphine-naloxone, buprenorphine, buprenorphine SL, opioid dependence, opioid conversion, opioid-induced hyperalgesia, analgesia, full agonist opioids, opioid tolerance.

Pain Physician 2012; 15:ES59-ES66 
A Ithough opioid medications have been used therapeutically for the relief of pain for thousands of years, in the 1940s opioids were restricted so that they could only be used legally when they were prescribed by a physician (1). At that time physicians were, and still are, legally and ethically responsible for the patients they treat with these agents and are liable to lose their medical licenses and risk criminal prosecution if they inappropriately prescribe these drugs (1). An ethical dilemma has arisen for physicians treating patients with opioids for chronic pain, balancing adequate pain control and quality of life with the risk of tolerance and chemical dependence on opioid drugs.

Chronic pain consists of long-acting, localized or diffuse complaints of discomfort and pain that have resisted more conservative care and persisted beyond the expected healing time (2). The treatment of chronic pain is difficult and associated with high economic and psychological cost (2). The use of opioids for chronic pain is gaining acceptance. Evidence supports that opioids can be safely administered in patients experiencing chronic pain without the development of chemical dependency and addiction (3). Despite the increasing use of these medications for chronic pain, concerns remain about physical dependence, adverse side effects, and the need for dose escalation to overcome tolerance in addition to issues surrounding prescription abuse (4). Recent evidence suggests that opioids are responsible for another problem that may limit their usefulness: opioid-induced hyperalgesia $(\mathrm{OIH})(4,5,6)$. Most broadly defined, OlH is the state of nociceptive sensitization caused by exposure to opioids $(4,7)$. This is characterized by a paradoxical response whereby a patient receiving opioids for the treatment of pain may actually become more sensitive to certain painful stimuli. Many

Table 1. Examples of affinity for opioid receptors of a pure opioid receptor agonist (morphine), mixed agonist-antagonists (buprenorphine, pentazocine), and opioid receptor antagonist (naloxone). In this table $+=$ agonist activity, $P=$ partial agonist/antagonist activity, - = antagonist activity, and $0=$ minimal to no receptor binding.

\begin{tabular}{|l|c|c|c|}
\hline \multicolumn{1}{|c|}{ Drug } & Mu & Delta & Kappa \\
\hline Morphine & + & 0 & + \\
\hline Buprenorphine & $\mathrm{P}$ & 0 & - \\
\hline Pentazocine & $\mathrm{P}$ & 0 & + \\
\hline Naloxone & - & - & - \\
\hline
\end{tabular}

biochemical mechanisms have been suggested as the cause of opioid-induced hyperalgesia $(3,6,7)$. There are currently no approved drugs for $\mathrm{OIH}$, and it remains a clinically unmet area of need.

There are many properties of buprenorphinenaloxone (buprenorphine $\mathrm{SL}$ ) that make it advantageous for the treatment of opioid dependence $(2,8)$. Buprenorphine SL is classified as a partial mu-agonist and kappa-antagonist. It has a high affinity for the muopioid receptor and a long duration of action. Table 1 lists sample opioids and their receptor affinities.

Buprenorphine is a partial agonist; its effects plateau at higher doses, limiting respiratory depression. (9) The unique pharmacologic profile of buprenorphine results in a low level of physical dependence and only mild withdrawal symptoms lasting one to 2 weeks upon cessation after prolonged administration. (10) In low doses, buprenorphine SL has an analgesic potency 25 to 50 times higher than an equivalent dose of morphine (11). Buprenorphine has been noted to be safe for use in elderly populations and patients with renal impairment. In addition, buprenorphine does not affect sex hormones or the immune system as morphine does (12). The Drug Enforcement Administration (DEA) has acknowledged the legality of an off-label use to treat pain with a sublingual buprenorphine preparation (13).

Figure 1 shows the chemical structure of buprenorphine, a semisynthetic phenanthrene which is derived from thebaine. Buprenorphine is a chemical which is highly lipophilic and predominantly protein bound $(96 \%)$ in systemic circulation (8).

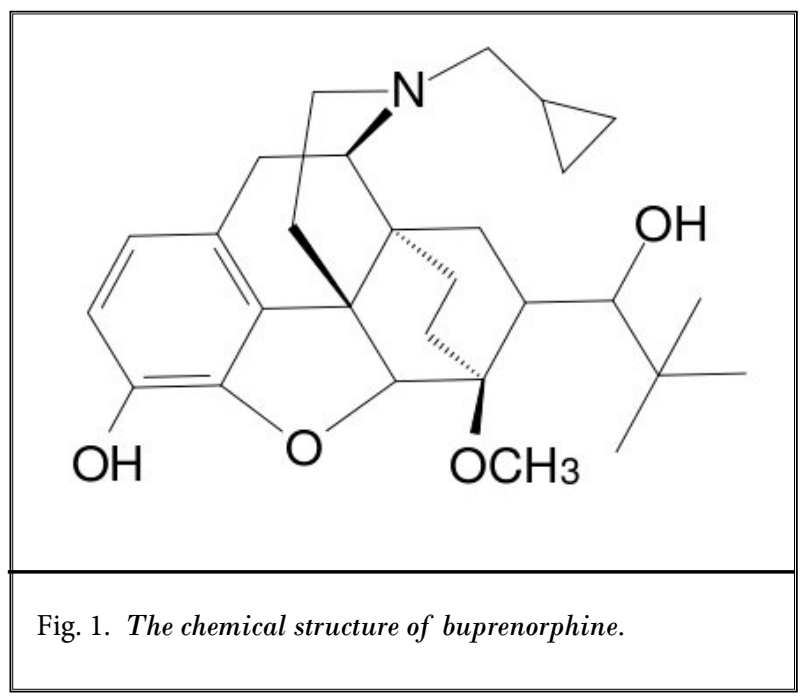


Aberrant opioid-seeking behavior may complicate the clinical picture of failed opioid therapy, and is often a manifestation of addiction and noncompliance. Noncompliance exists in many forms, including: unexpected results on toxicologic screening; frequent requests for dose increases; concurrent use of nonprescribed psychoactive substances; failure to follow the dosage schedule; failure to adhere to concurrently recommended treatments; frequently reported loss of prescriptions or medications; frequent visits to the emergency room for opioid therapy; missed follow-up visits; frequent extra appointments at the clinic or office; prescriptions obtained from other providers; and tampering with prescriptions (14). When a patient displays one or many of these behaviors, the clinician is often left with few options to continue treating such a patient. This type of patient may not be an acceptable candidate for traditional opioid therapy, yet still requires treatment for escalating pain. In this situation the prescriber is left with few options to adequately treat the patient appropriately.

In the 1970s a parenteral buprenorphine dosage form indicated for pain treatment was brought to the American market. Since that time, a sublingual preparation alone and in combination with naloxone have become available. This product is a Schedule III, FDAapproved drug for treatment of opioid dependency. Buprenorphine $S L$ tablets have been suggested as useful for treatment of opioid-induced hyperalgesia (5). The analgesia provided by sublingual buprenorphine in chronic pain patients must be quantitatively valuated in order to predict patient response to this treatment. The purpose of this study was to evaluate the effectiveness of conversion to buprenorphine $S L$ in tablet form for patients with high levels of persistent pain on full agonist opioid medications, and to identify demographic groups most likely to benefit from therapy with this agent. To the best of our knowledge, no one has reported the use of buprenorphine SL for pain management of patients with chronic pain on high-dose opioid medications.

\section{Methods}

This was a single-center study of chronic pain patients who had experienced continued or worsening pain despite the use of short- and long-acting opioid analgesics. Patients were assessed from their history, a physical examination, and urine testing for toxicology prior to initiating treatment. From December 2007 through July 2010 patients were considered for conversion from opioid drugs, based on their high dose or ineffective use of these drugs, to sublingual buprenor- phine SL. Overall, 104 patients were converted from opioid medications, continued buprenorphine SL for more than 60 days, and were included in this study. All patients gave informed consent prior to conversion to buprenorphine SL tablets.

Prior to conversion to buprenorphine SL, patients underwent an education program in the office with a nurse practitioner. In the program, patients were thoroughly instructed on the use, risks, and benefits of buprenorphine SL. Patients filled out a questionnaire ascertaining their current Quality of Life (QoL scale) and pain level. Patient levels of withdrawal were evaluated with their Clinical Opiate Withdrawal Scale (COWS) score.

Sublingual buprenorphine-naloxone in tablet form was prescribed to patients with poorly controlled chronic pain. Patients were on a variety of opioid medications. Some were on different types of long-acting and short-acting opioid medications. The medications were converted to morphine equivalents and added together to obtain a preinduction amount of morphine equivalents for each patient.

Buprenorphine SL was administered to patients after they had discontinued all opioid medications at least 24 hours prior (48-72 hours for methadone). Initially, $8 \mathrm{mg}$ of buprenorphine SL was given sublingually. Patients were instructed to dissolve an additional dose of $8 \mathrm{mg}$ one hour later if pain or withdrawal symptoms continued. Patients were instructed not to exceed 32 $\mathrm{mg}$ of buprenorphine SL daily.

After one week, the dosing of buprenorphine SL was titrated up or down based on each patient's report of opioid abstinence symptoms and pain complaints. Patients were then evaluated at least monthly. The pain level and QoL scale were determined at each visit.

All data were collected from patient electronic medical records in a standardized blinded manner. The patient's age, sex, preinduction medication, morphine equivalent dosage, and urine screening results were recorded. Table 2 shows the equianalgesic conversion doses of opioids utilized in the study.

Further, it was determined if patients continued to take buprenorphine SL or whether they switched back to previous opioid drugs. All patients who had prescriptions for buprenorphine SL were evaluated for inclusion in the study. Patients were disqualified for the study if they were not on buprenorphine SL for at least 60 days, were taking buprenorphine SL before the first clinic visit, were noncompliant in the treatment plan, or continued to use opioid medications. The primary outcome evaluated was reduction in self-reported pain 
Table 2. Equianalgesic dosage morphine equivalent conversion table.

\begin{tabular}{|c|c|}
\hline DRUG & DOSE \\
\hline Morphine & $30 \mathrm{mg}$ \\
\hline Hydrocodone & $30 \mathrm{mg}$ \\
\hline Fentanyl patch & $10 \mathrm{mcg}$ \\
\hline Methadone & $7.5 \mathrm{mg}$ \\
\hline Oxycodone & $20 \mathrm{mg}$ \\
\hline Oxymorphone & $10 \mathrm{mg}$ \\
\hline
\end{tabular}

Table 3. Patient demographics and preinduction morphine dose.

\begin{tabular}{|l|c|c|c|}
\hline Parameter & Percent & Mean & Range \\
\hline Male & $58 \%$ & & \\
\hline Female & $42 \%$ & & \\
\hline Age (year) & & $49 \pm 12$ & $21-78$ \\
\hline Duration (months) & & $10.3 \pm 6$ & $2-42$ \\
\hline $\begin{array}{l}\text { Preinduction } \\
\text { morphine (mg) }\end{array}$ & & $180 \pm 160$ & $10-840$ \\
\hline
\end{tabular}

after conversion to buprenorphine SL using a standard 11 point scale (0-10). The secondary outcome analyzed was a change in the QoL scale.

\section{Results}

Of the 104 patients included in the final data analysis, $58 \%$ were men and $42 \%$ were women. Their average age was 49 years with a range of 21-78 years. Patients on average continued buprenorphine SL tablets for 10.3 months by the end of the study. The mean daily preinduction morphine equivalent dose of opioid was $180 \mathrm{mg}$ (Table 3).

The preinduction prevalence of opioid medication prior to conversion is presented in Fig. 2. The most common principal opioid drug used before conversion to buprenorphine SL was oxycodone, with 47 patients $(45 \%)$ in this group. There were 15 patients primarily using fentanyl, 14 using hydrocodone, 11 using methadone, 10 using oxymorphone, and 7 using morphine. It was common for patients to be using long-acting and short-acting opioids concurrently.

Figure 3 shows the preinduction morphine equivalent and the change in pain score after buprenorphine SL conversion. Overall, patients tolerated sublingual buprenorphine therapy very well and reported few side effects. Patients who were switched from traditional

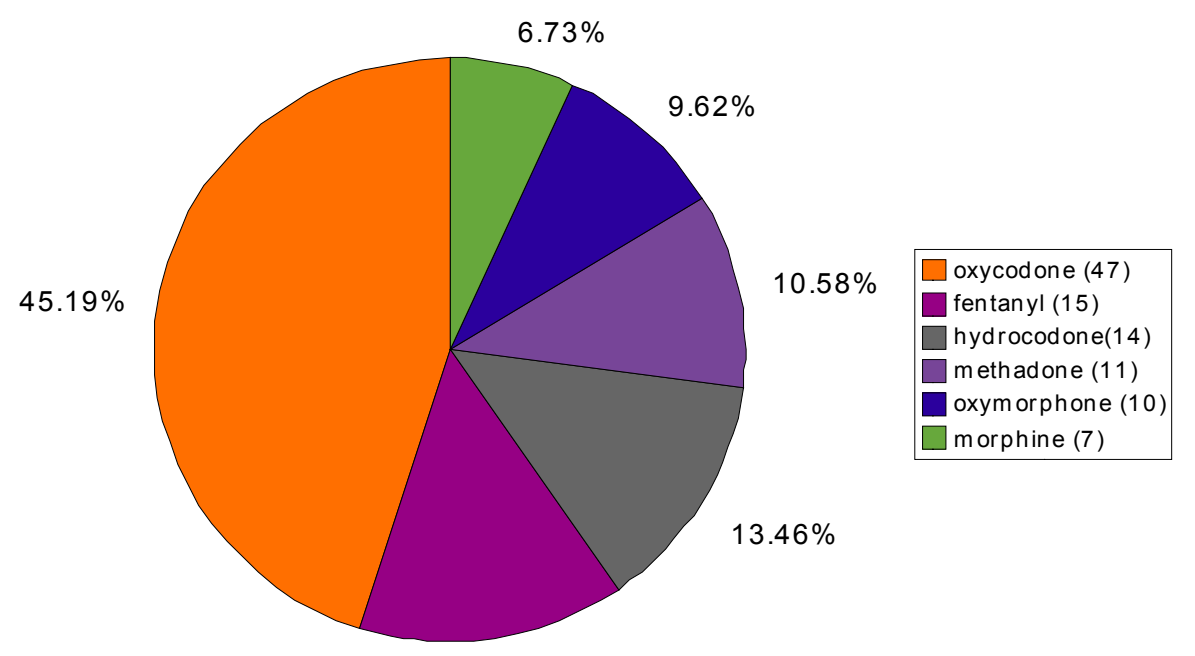

$14.42 \%$

Fig. 2. Preinduction opioid drug prevalence. 

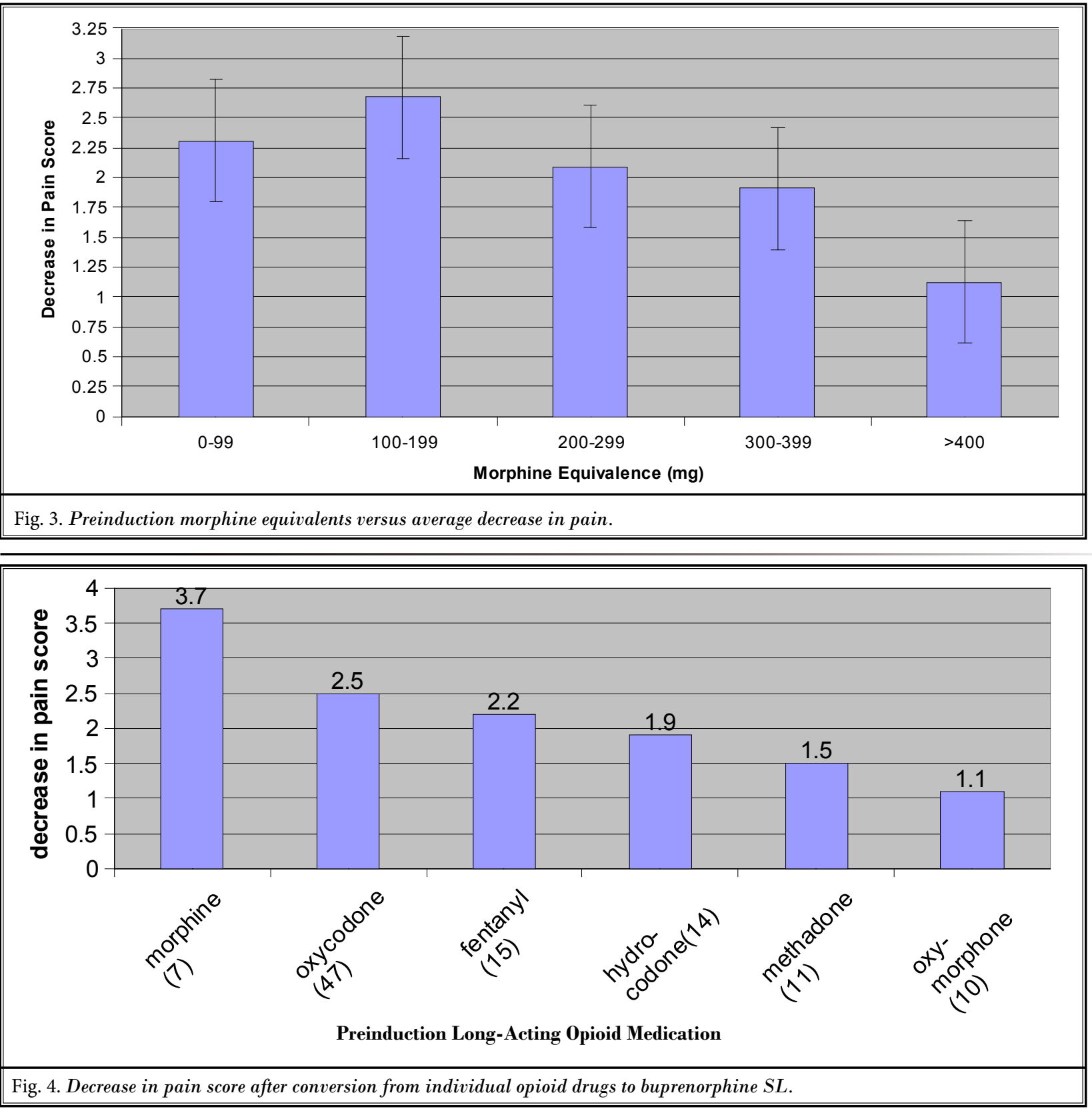

opioids to buprenorphine SL and continued therapy for at least 60 days experienced a 2.3 point $(P<0.001)$ reduction in pain. The difference in pain scores at baseline and after conversion to buprenorphine SL therapy was statistically and clinically significant. The patientreported change QoL score after buprenorphine SL induction failed to reach statistical significance $(P=0.14)$.

Patients taking between $100-199$ mg morphine equivalent per day experienced the greatest decrease in pain score, on average 2.7 points. Patients taking more than $400 \mathrm{mg}$ morphine equivalent per day experienced only a 1.1 point decrease in pain score, providing the smallest analgesic effect in this group of patients. Patients taking 200 - $299 \mathrm{mg}$ morphine equivalent all experienced a more than 2 point decrease in pain scores postinduction.

The preinduction opioid drug that a patient was switched from had an effect on the patient's reported postinduction pain score (Fig. 4). Patients that were 
switched from primarily morphine to buprenorphine SL had the greatest reduction in pain score and decreased 3.7 points on average. Patients taking oxycodone, fentanyl, and hydrocodone all had appreciable decreases in pain of 2.5,2.2, and 1.9 respectively. Those switched from methadone or oxymorphone exhibited the smallest clinical change in pain score, namely, a 1.5 point decrease with methadone, and a 1.1 point decrease in pain score on average with oxymorphone.

\section{Discussion}

Pain clinicians routinely are faced with patients who initially arrive on high dose opioid medication prescribed to them by other providers. This leads to the decision whether to continue them on high dose pain medications, try opioid rotation with an alternate opioid medication, titrate them down to lower doses, consider alternate analgesic treatments such as a spinal cord stimulator or spinal infusion pump, send them for inpatient detoxification, or choose an alternate pain medication such as buprenorphine SL. To the best of our knowledge, based on the results of this study, and since no previous studies have addressed this issue, clinicians should consider conversion to sublingual buprenorphine for patients who present on high doses of opioid medication.

Similarly, clinicians will also have to deal with their own patients who over time need escalating doses of opioid medications. This may be either opioid tolerance or may be opioid-induced hyperalgesia. These patients appear to respond well to conversion to sublingual buprenorphine as well.

We offered buprenorphine SL tablet therapy to 273 patients. Many refused to participate in the conversion. Some who refused were new patients looking for only high-dose opioid medication. Some were offered buprenorphine SL because they had illicit substances in their urine, and we refused to continue their usual opioid medications. To the best of our knowledge, both of these types of patients were unlikely to return to our clinic for either follow-up visits or buprenorphine conversion.

Results from our retrospective data collection and analysis indicate that buprenorphine SL tablets can be an effective analgesic for patients who have not sustained successful analgesia with traditional opioid medications. Patients who converted to sublingual buprenorphine and continued therapy for at least 60 days experienced a 2.3 point decrease in pain scores. This decrease in pain scores did not, however, correlate with an increase in patient reported QoL. It is not clear why there was a lack of correlation between reduction in pain score and QoL improvement but this might be due individualized nature of pain.

This study looked at preinduction doses of opioids in morphine equivalents as well as the type of opioid medications the patients were taking. Patients taking a dosage of $0-299 \mathrm{mg} / \mathrm{d}$ morphine equivalent before induction experienced, on average, a decrease in pain of more than 2 points. Patients taking a dosage of $100-$ $199 \mathrm{mg} / \mathrm{d}$ morphine equivalent before induction experienced the greatest level of analgesia, resulting in a 2.7 point decrease in pain score. Patients taking a dosage of more than $400 \mathrm{mg} / \mathrm{d}$ morphine equivalent experienced the lowest decrease in pain on average. However, all dosage ranges of morphine equivalents noted improvements in pain scores on sublingual buprenorphine.

The principal preinduction opioid drug the patient was maintained on may also affect the decision of a clinician to use buprenorphine SL. Patients taking morphine, oxycodone, and fentanyl appear to be the best candidates for a conversion to buprenorphine SL. Patients using these medications experienced a decrease of greater than 2 points on average. Those specifically maintained on morphine reported a decrease of 3.7 points in pain on average. This dramatic decrease in pain with patients on morphine leads us to wonder specifically if opioid hyperalgesia is present in this category of patients on high dose morphine for extended periods of time.

Patients taking methadone or oxymorphone before conversion to buprenorphine SL experienced the smallest difference in analgesia after switching. After conversion, reported pain scores for patients taking methadone decreased 1.5 points and oxymorphone patients decreased 1.1 points. It is not clear why patients who were taking oxycodone would experience a very robust decrease in pain of 2.5 points, while patients taking oxymorphone, an active metabolite of oxycodone, did not experience a similar analgesic response. Based on other well accepted publications, the reduction in pain intensity that was noted in our study was clinically meaningful. Farrar and colleagues (15), using a database containing information from more than 2,700 patients in 10 placebo-controlled analgesic trials involving pregabalin, sought to examine the relationship between a numerical rating scale of pain intensity and a patient's global impression of change during the course of the trial. Based on the outcome of this research, a 2 point decrease, or $30 \%$ reduction, represents a clinically 
meaningful improvement in a patient's pain (15).

Once patients were converted to buprenorphine and established an effective dose, no escalation in medication use was noted. This is similar to results noted in a European study of transdermal buprenorphine (16). Patients did not overuse medication and rarely ran out early. The long half-life of buprenorphine may explain this. Since it only partially stimulates the opioid receptor, there appears to be less tolerance. Patients generally felt less sedated and noted improved cognition. The side effects most often recorded that were attributed to buprenorphine SL within the clinic were nausea, dizziness, urinary retention, and sexual dysfunction.

According to the package insert, the most common adverse effects during 4-week clinical trials of buprenorphine SL16 mg daily were headache (36.4\%), withdrawal symptoms $(25.2 \%)$, generalized pain (22.4\%), nausea/vomiting (15\%/7.5\%), insomnia (14\%), sweating or diaphoresis (14\%), constipation (12.1\%), and abdominal pain (11.2\%). Less common adverse reactions include vasodilation $(9.3 \%)$, chills $(7.5 \%)$, asthenia $(6.5 \%)$, infection $(5.6 \%)$, rhinitis $(4.7 \%)$, diarrhea $(3.7 \%)$, and back pain (3.7\%) (17).

One can surmise that many of these patients who were successfully converted over to buprenorphine SL were probably exhibiting at least opioid tolerance, if not opioid-induced hyperalgesia. Conversion to buprenorphine SL improved analgesia in the vast majority of these patients. Buprenorphine is suggested to be specifically anti-hyperalgesic in humans (18). However, recent data in animal studies indicate that buprenorphine can induce hyperalgesia. (19). Nevertheless, it is likely that the anti-hyperalgesic nature unique pharmacology of buprenorphine contributed to the efficacy in many of these patients who may have been opioid hyperalgesic. While studies have not proven $\mathrm{OIH}$ as a unique and defined phenomenon, the concept is seen in clinical practice: high doses of traditional opioid medications, such as morphine and oxycodone, result in tolerance and decreased analgesia (20). Buprenorphine provides an alternative for clinicians struggling with these patient issues.

Results of efficacy have been found in European studies with transdermal buprenorphine as well. One study found that $90 \%$ of patients with cancer and noncancer pain had excellent analgesia with long-term use of transdermal buprenorphine (21).

One of the weaknesses of the study is that it was an observational chart review with no control group. Chart reviews are advantageous in that the data is eas-
Table 4. The most common adverse effects during the 4-week clinical trial using buprenorphine $16 \mathrm{mg}$ daily (17).

\begin{tabular}{|l|c|l|c|}
\hline \hline Headache & $36.4 \%$ & Vasodilation & $9.3 \%$ \\
\hline Withdrawal Symptoms & $25.2 \%$ & Vomiting & $7.5 \%$ \\
\hline Generalized Pain & $22.4 \%$ & Chills & $7.5 \%$ \\
\hline Nausea & $15 \%$ & Asthenia & $6.5 \%$ \\
\hline Insomnia & $14 \%$ & Infection & $5.6 \%$ \\
\hline Sweating Or Diaphoresis & $14 \%$ & Rhinitis & $4.7 \%$ \\
\hline Constipation & $12.1 \%$ & Diarrhea & $3.7 \%$ \\
\hline Abdominal Pain & $11.2 \%$ & Back Pain & $3.7 \%$ \\
\hline
\end{tabular}

ily accessible and sample sizes can be high. This type of study is especially useful in identifying trends which can be examined in subsequent randomized controlled trials. Unfortunately, this type of study is limited because patient charts may be incomplete, missing, or unrecoverable; there may be difficulty interpreting information in patient charts; verification of information may be difficult as events happened in the past; and cause and effect cannot be established as in a randomized controlled trial (22). The retrospective nature of this study cannot measure certain key statistics and bias may have affected the patient population selected. Questions raised as a result of this initial investigation should fuel future prospective research with a refined study design and provide guidance to clinicians making treatment decisions in this patient population.

\section{Conclusion}

Buprenorphine SL in tablet form is an effective analgesic in patients who have failed or become tolerant to traditional opioid therapy for chronic pain. Sublingual buprenorphine's unique pharmacology as a partial mu-agonist results in unique therapeutic effects. Additionally, patients taking buprenorphine SL do not appear to exhibit hyperalgesia and tolerance to the medication.

When patients presenting with chronic pain are taking high dose opioid drugs and experiencing tolerance, it represents a major ethical and treatment dilemma for the practitioner. Preventing medication overuse and escalation, while treating the underlying pain, is challenging in such patients. Buprenorphine SL represents a viable and efficacious treatment for many of these patients. Further research is needed to identify which demographic groups are most likely to benefit from conversion from traditional opioid drugs to buprenorphine SL. In addi- 
tion, further investigation of changes in patient quality of life during buprenorphine SL therapy should evaluate the effects on family relationships, mental clarity, and physical functioning. Buprenorphine SL tablets represent a safe and useful tool for office-based conversion of difficult or high-risk chronic opioid patients within a traditional pain practice.

\section{Acknowledgments}

The authors would like to thank the editorial board of Pain Physician for review and criticism in improving the manuscript.

\section{Author Affilations}

Dr. Daitch is Clinical Director of Advanced Pain Management and Spine Specialists, and Medical Direc- tor of Park Center for Procedures ASC. Dr. Frey is Associate Professor at Virginia Commonwealth University, Director of Interventional Physiatry at Advanced Pain Management and Spine Specialists. David Silver is a Staff Pharmacist at Lee Memorial Hospital, Fort Myers, FL. Carol Mitnick is an ARNP at Advanced Pain Management and Spine Specialists. Danielle Daitch is a second year medical school student at Thomas Jefferson Medical School, Philadelphia, PA. Dr. Pergolizzi is an adjunct faculty member, Department of Anesthesiology, Georgetown University Medical School; Adjunct Assistant Professor, Department of Medicine, Johns Hopkins University School of Medicine, Baltimore, MD Associate Professor, Department of Pharmacology, Temple University School of Medicine, Philadelphia, PA.

\section{References}

1. Ballantyne JC, Mao, J. Opioid therapy for chronic pain. N Engl J Med 2003; 349:1943-1953.

2. Malinoff, HL, Barkin, RL, Wilson, G. Sublingual buprenorphine is effective in the treatment of chronic pain syndrome. Am J Ther 2005; 12:379-384

3. Lee M, Silverman, SM, Hansen $\mathrm{H}, \mathrm{Pa}$ tel VB, Manchikanti L. A comprehensive review of opioid-induced hyperalgesia. Pain Physician 2011; 14:145-161.

4. Chu, LF, Angst, MS, Clark, D. Opioid-induced hyperalgesia in humans: Molecular mechanisms and clinical considerations. Clin J Pain 2008; 24:479-496.

5. Angst, MS, Clark, JD. Opiod-induced hyperalgesia: A qualitative systematic review. Anesthesiology 2006; 104:570-587.

6. Mercadante, S, Ferrera P, Villari P, Arcuri E. Hyperalgesia: An emerging iatrogenic syndrome. J Pain Symptom Manage 2003; 26:769-775.

7. Mao J. Opioid-induced abnormal pain sensitivity: Implications in clinical opioid therapy. Pain 2002; 100:213-217.

8. Johnson RE, Fudala PJ, Payne R. Buprenorphine: considerations for pain management. J Pain Symptom Manage 2005; 29:297-326.

9. Julien R. A Primer of Drug Action. Worth Publishers, New York, NY, 2008.
10. Fudala PJ, Jaffe JH, Dax EM, Johnson RE. Use of buprenorphine in the treatment of opioid addiction. II. Physiologic and behavioral effects of daily and alternateday administration and abrupt withdrawal. Clin Pharmacol Ther 1990;47:525534 .

11. Gutstein H, Akil H. Opioid analgesics. In: Brunton LB, Lazo JS, Parker KL, (eds). Goodman \& Gilman's The Pharmacological Basis of Therapeutics. 11th ed. McGraw-Hill, New York, NY, 2006, pp 547590.

12. Pergolizzi J, Aloisi AM, Dahan A, Filitz J, Langford R, Likar R, Mercandante $S$, Morlion B, Raffa RB, Sabatowski R, Sacerdote P, Torres LM, Weinbroum AA. Current knowledge of buprenorphine and its unique pharmacological profile. Pain Pract 2010; 10:428-450.

13. Heit HA, Covington E, Good PM. Dear DEA. Pain Med 2004; 5:303-308.

14. Fishman SM, Wilsey B, Yang J, Reisfield $G$, Bandman TB, Borsook D. Adherence monitoring and drug surveillance in chronic opioid therapy. J Pain Symptom Manage 2000; 20:293-307.

15. Farrar JT, Young JP Jr, LaMoreaux L, Werth JL, Poole RM. Clinical importance of changes in chronic pain intensity measured on an 11-point numerical pain rating scale. Pain 2001; 94:149-158.
16. Griessinger N, Sittl R, Likar R. Transdermal buprenorphine in clinical practice a post-marketing surveillance study in 13,179 patients. Curr Med Res Opin 2005; 21:1147-1156.

17. Suboxone [package insert]. Richmond, VA: Reckitt-Benckiser; 2010.

18. Koppert W, Ihmsen H, Korber N, Wehrfritz A, Sittl R, Schmelz M, Schüttler J. Different profiles of buprenorphine-induced analgesia and antihyperalgesia in a human pain model. Pain 2005; 118:1522.

19. Wala EP, Holtman JR Jr. Buprenorphineinduced hyperalgesia in the rat. Eur J Pharmacol 2011; 651:89-95.

20. Chapman CR, Davis J, Donaldson GW, Naylor J, Winchester D. Postoperative pain trajectories in chronic pain patients undergoing surgery: The effects of chronic opioid pharmacotherapy on acute pain. J Pain 2011. Epub ahead of print.

22. Likar R, Kayser H, Sittl R. Long-term management of chronic pain with transdermal buprenorphine: A multicenter, open-label, follow-up study in patients from three short-term clinical trials. Clin Ther 2006; 28:943-952.

22. Hess DR. Retrospective studies and chart reviews. Respir Care 2004; 49: 11711174 . 